\title{
Corn silk polysaccharide ameliorates high fat diet induced hepatic steatosis in mice
}

\author{
Wang Tailin ${ }^{1}$, Wang Zhiwen ${ }^{2,3}$, Liu $\mathrm{Yi}^{2}$ and Huang $\mathrm{Li}^{2, *}$ \\ ${ }^{1}$ Changsha Health Vocational College, Changsha 410000, Hunan, China \\ ${ }^{2}$ Yueyang Key Laboratory of Chronic Noncommunicable Diseases, Yueyang Vocational \& Technical College, Yueyang 414000, Hunan, \\ China \\ ${ }^{3}$ College of Life Science and Health, Wuhan University of Science and Technology, Wuhan 430065, Hubei, China
}

\begin{abstract}
To study the therapeutic effect of corn silk polysaccharide (CSP) on NAFLD mice induced by high fat diet. C57BL/6J mice were divided into normal control group (NC), high fat diet (HFD) group, HFD $+200 \mathrm{mg} / \mathrm{kg} \mathrm{CSP}$ group, and HFD $+600 \mathrm{mg} / \mathrm{kg}$ CSP group. NAFLD mouse model was established by HFD feeding. Blood and liver tissues of each group were collected and biochemical and pathological tests were performed. The energy intake of NAFLD model group was higher than that of normal control group, and the food intake, water intake, and excretion of NAFLD model group were lower than that of normal control group. There was no statistical significance in the food intake, energy intake, water intake, and excretion of CSP group compared with that of NAFLD model group, nor was there any statistical significance between CSP and two doses of CSP. Biochemical tests showed that CSP decreased the levels of alanine aminotransferase, aspartate aminotransferase, triglyceride and total cholesterol in serum of HFDfed mice, and inhibited the expressions of IL- 6 and TNF- $\alpha$ in liver tissue. Pathological results showed that CSP improved HFD-induced hepatic steatosis.
\end{abstract}

\section{Introduction}

Fatty liver is divided into alcoholic fatty liver disease and nonalcoholic fatty liver disease (NAFLD) ${ }^{[1]}$. Among them, NAFLD is closely related to metabolic diseases such as obesity, insulin resistance, hypertension and dyslipidemia, so NAFLD belongs to chronic metabolic abnormal liver disease ${ }^{[2]}$. The prominent feature of NAFLD is the persistent pathological changes in the course of disease. Hepatocellular steatosis caused by metabolic disorders of the body at the beginning (exclusion of clear hepatic damage factors) developed into non-alcoholic steatohepatitis, followed by liver fibrosis and cirrhosis, and even eventually may lead to hepatocellular carcinoma. Therefore, NAFLD is a potential factor for the development of primary hepatocellular carcinoma ${ }^{[3]}$. Moreover, NAFLD affects the physical health of about $30 \%$ of adults worldwide ${ }^{[4]}$. Therefore, NAFLD has become a public health problem closely related to our life. Although many strategies have been developed for the treatment of NAFLD, there are currently no drugs specific for NAFLD.

Corn silk polysaccharide (CSP) is an effective component extracted from corn whisker, a traditional Chinese medicine, which has tumor inhibition, hypoglycemia, diuresis and antioxidant effects ${ }^{[5-7]}$. In recent years, more and more studies have been done on its free radical scavenging and antioxidant effects. After 30 days of ingastric administration, the oxidative stress indexes in brain, liver and serum of the aged rats were significantly improved, and the ability of anti-oxidation and anti-aging was enhanced. Based on the fact that NAFLD is a stress and inflammatory liver injury caused by lipid metabolism disorders in hepatocytes and the pharmacological effect of CSP [5-8], it is speculated that CSP may have an anti-NAFLD effect, while the effect and mechanism of CSP on NAFLD have not been studied yet. Therefore, this study aims to explore whether CSP has a protective effect on the liver in NAFLD and its mechanism.

\section{Materials and methods}

\subsection{Preparation of CSP}

Corn silk was bought in Anhui Bozhou Chinese medicinal materials wholesale market, after cleaning, drying, crushing reserve. The corn silk powder was boiled in hot water for $2 \mathrm{~h}$, and the supernatant was separated by centrifugation at $3000 \mathrm{r} / \mathrm{min}$. The corn silk residue was extracted again by the same method, and the supernatant was combined twice. The protein was removed by SEVAGE method after the decolorization with macroporous resin. Dried until powder ready, dissolved in normal saline when used.

\footnotetext{
* Corresponding author: 40983986@qq.com
} 


\subsection{Animal management}

SPF grade 8-week-old male C57BL/6J mice were purchased from Beijing Vitong Lihua Experimental Animal Technology Co., Ltd., and reared in SPF grade animal house (no pathogen condition; 12h light/dark cycle; The room temperature is maintained at $23-25{ }^{\circ} \mathrm{C}$; Humidity was maintained at $55-65 \%$ ), and the mice were given a free diet and water before being given a special diet. Animal testing programs follow the ethical guidelines for laboratory animals.

\subsection{Mice model of NAFLD}

NAFLD mouse model was established by high fat diet (HFD) induction. The mice were randomly divided into normal control group (NC), HFD group, HFD+200 $\mathrm{mg} / \mathrm{kg}$ CSP group and HFD+600 $\mathrm{mg} / \mathrm{kg}$ CSP group, with 10 mice in each group. Mice in NC group were fed with normal diet for $10 \mathrm{wk}$; HFD group, HFD+200 $\mathrm{mg} / \mathrm{kg}$ and HFD $+600 \mathrm{mg} / \mathrm{kg}$ CSP group were fed HFD diet $(60 \%$ normal diet, $35 \%$ fat and $5 \%$ cholesterol) for $10 \mathrm{wk}$. HFD+200 mg/kg CSP group and HFD+600 mg/kg mice in the CSP group were intragastrically given $200 \mathrm{mg} / \mathrm{kg}$ and $800 \mathrm{mg} / \mathrm{kg}$ CSP every day during the 7-10 weeks of feeding, respectively. Mice in the NC group and the HFD group were intragastrically given the same volume of normal saline during the same period. At the end of the 10th week, the mice were weighed, anesthetized and killed. The head was severed for blood collection, and the liver was dissected and weighed.

\subsection{Blood biochemical test}

Blood samples were placed in a vacuum anticoagulant tube for standing for $10 \mathrm{~min}$, and centrifuged at 3000 $\mathrm{r} / \mathrm{min}$ at room temperature for $20 \mathrm{~min}$ to collect serum. Then the serum alanine transaminase (ALT), aspartate aminotransferase (AST), total triglyceride (TG) and total cholesterol (TC), low density lipoprotein cholesterol (LDL-C) and high density lipoprotein cholesterol (HDLC) levels were analyzed by automatic biochemical analyzer.

\subsection{H\&E staining}

The liver tissues were soaked overnight in 4\% formaldehyde solution, then embedded and fixed in paraffin, and liver sections were prepared at $5 \mu \mathrm{m}$. After routine dewaxing and rehydration of the liver sections, $\mathrm{H} \& \mathrm{E}$ staining was performed, and the pathological changes of the liver were observed under a microscope and photographed.

\subsection{Oil-red O staining}

The liver tissue preserved with special embedding agent for frozen sections was made into sections $10 \mu \mathrm{m}$ thick sections, washed with distilled water and rinsed with $60 \%$ isopropanol for 20-30 s. The oil-red O sulution was dropped and incubated at room temperature for $10 \mathrm{~min}$.
The excess staining solution was slightly washed with $60 \%$ isopropanol, then washed with distilled water to remove isopropanol, and the nucleus was stained with hematoxylin and differentiated with $1 \%$ hydrochloric acid-alcohol solution. Finally, they were rinsed with water for $10 \mathrm{~min}$ and dried at room temperature. Lipid accumulation in liver tissue was observed under a microscope and photographed.

\subsection{ELISA test}

After the liver tissues were homogenized with RIPA solution, the homogenate was collected and the protein was quantified by BCA method. The liver tissue homogenate was taken and detected by ELISA according to the steps of the kit instructions. After the reaction, the absorbance value was measured at $450 \mathrm{~nm}$ with a 96well plate spectrophotometer. The standard curve was drawn according to the standard substance, and the contents of IL- 6 and TNF- $\alpha$ in unit mg protein were calculated.

\subsection{Measurement of TC and TG in liver tissue}

The liver tissue homogenate sample after protein quantification by BCA method was taken and reacted according to the steps in the kit instructions. After the reaction, the absorbance value was measured at the wavelength of $505 \mathrm{~nm}$ with a spectrophotometer, and the standard curve was drawn according to the standard substance to calculate the content of TG and TC in unit mg protein.

\subsection{Statistical analysis}

Data were expressed as Mean $\pm \mathrm{SD}$ and analyzed statistically by SPSS 19.0 software. One-way analysis of variance was used to compare the data. $P<0.05$ was considered statistically significant.

\section{Results}

\subsection{Comparison of serum lipid levels in mice}

The serum TG, TC and LDL-C in NAFLD model group were significantly higher than those in control group. The levels of TG, TC and LDL-C in the treatment group were significantly lower than those in the model group $(P<0.05)$, as shown in Fig. 1.

\subsection{CSP inhibited HFD-induced liver injury and histopathological changes}

The results (Fig. 2) showed that the serum ALT and AST activities in the HFD group were significantly increased $(P<0.01)$, while CSP treatment could significantly inhibit the HFD-induced ALT and AST activity increase $(P<0.05$ or $P<0.01)$. It is suggested that CSP can inhibit HFD-induced liver injury. The liver/body weight index was further calculated, and the results (Fig. 3) showed 
that compared with the NC group, the liver/body weight index in the HFD group was significantly increased $(P<0.01)$. Compared with HFD group, the liver/body weight index in $\mathrm{HFD}+200 \mathrm{mg} / \mathrm{kg}$ CSP group and HFD $+600 \mathrm{mg} / \mathrm{kg}$ CSP group was significantly decreased $(P<0.05)$. The pathological changes of liver were directly observed by H\&E staining (Fig. 4). The results showed that the liver tissue of the HFD group showed a large number of vacuol-like changes of liver cells accompanied by infiltration of inflammatory cells, while hepatological changes induced by HFD were significantly reduced in the CSP treatment group.

\subsection{CSP inhibits HFD-induced inflammatory response in liver tissue}

The expression levels of IL- 6 and TNF- $\alpha$ in liver tissue were detected by ELISA to assess the inflammatory response in liver. The results (Fig. 5) showed that the levels of IL- 6 and TNF- $\alpha$ in liver tissue were significantly increased in HFD group compared with the NC group $(P<0.01)$; Compared with HFD group, CSP could dose-dependentially reduce the levels of IL- 6 and TNF- $\alpha$ induced by HFD $(P<0.05$ or $P<0.01)$.

\subsection{CSP decreased hepatic lipid accumulation induced by HFD}

The results showed that the contents of TG and TC in serum of HFD group were significantly increased $(P<0.01)$, and CSP could significantly inhibit the increase of TG and TC content induced by HFD. This phenomenon was also observed in the liver tissue (Fig. 6 ). The observation results of oil red $\mathrm{O}$ staining showed that in the HFD group, due to the large amount of vacuolar steatosis in the liver tissue, the fat droplets conflued into large fat droplets, which accumulated in the liver cells in large quantities; CSP significantly improved lipid accumulation in hepatocytes of HFDinduced liver tissue. The lipid droplets were counted, and the results showed that the number of lipid droplets in liver tissue of HFD group was significantly increased, and CSP reduced HFD-induced lipid droplet accumulation in a concentration-dependent manner.
A

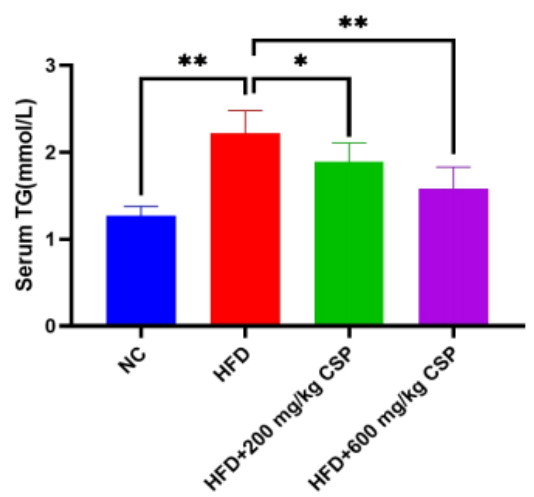

B

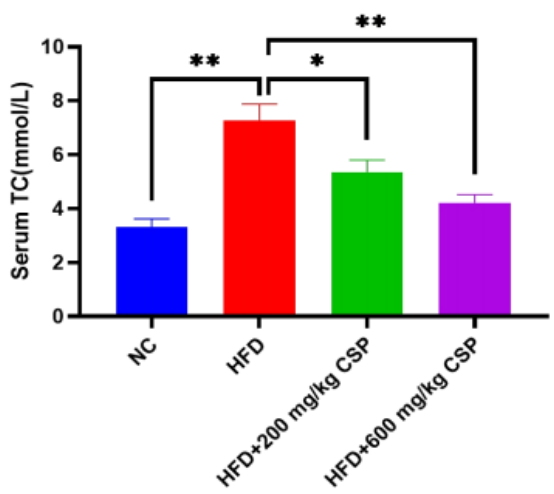

C

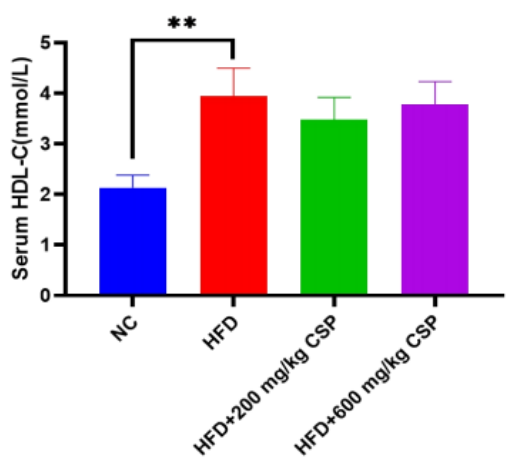

D

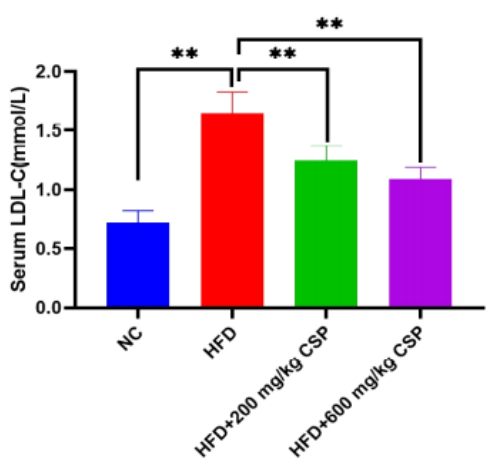

Fig. 1. The contents of TG (A), TC (B), HDL-C (C) and LDL$\mathrm{C}$ (D) in serum of each group. ${ }^{*} P<0.05,{ }^{*} P<0.01$ 
A

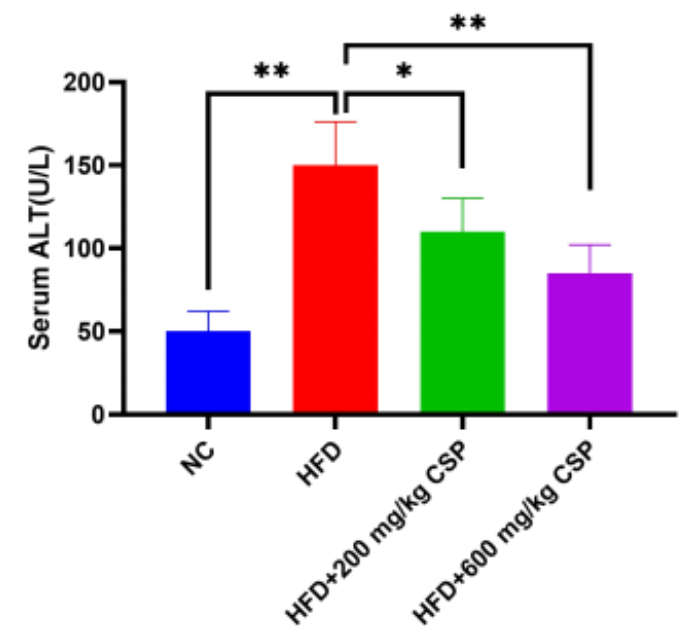

B

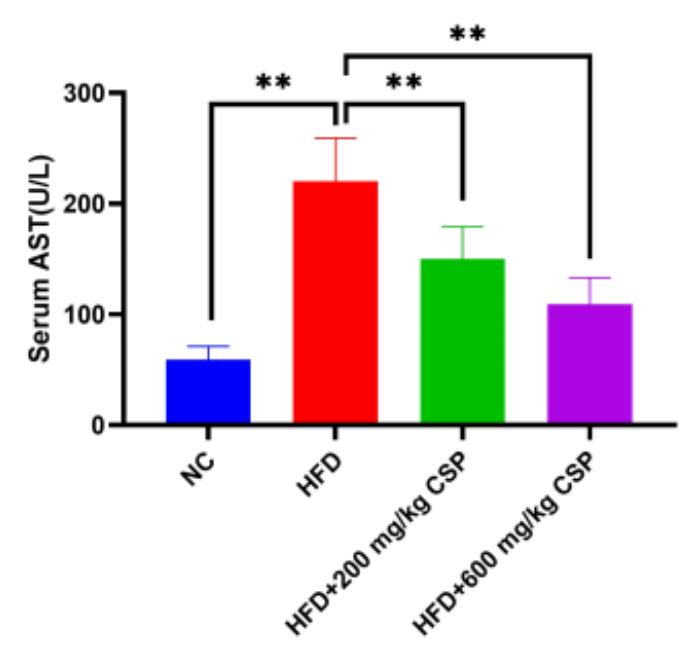

Fig. 2. The contents of ALT and AST in serum of each group. $* P<0.05,{ }^{*} * P<0.01$.

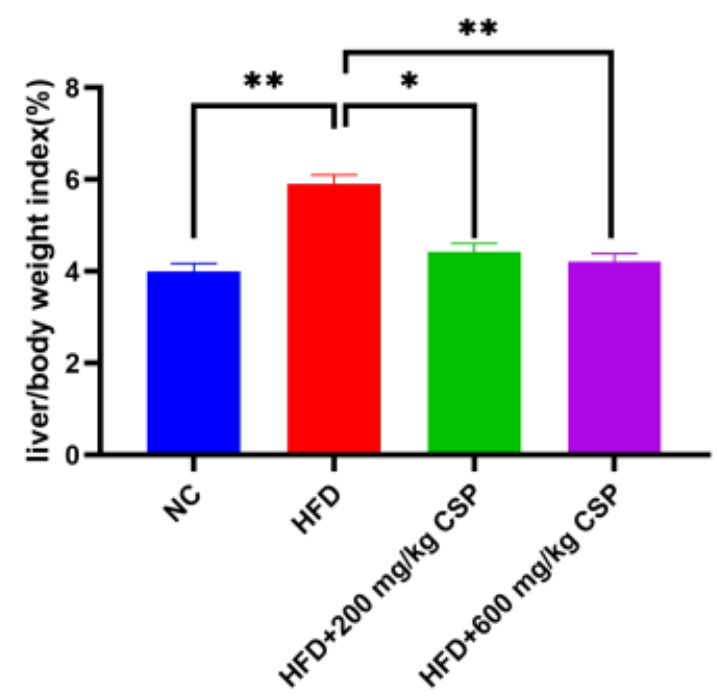

Fig. 3. The liver index of each group. ${ }^{*} P<0.05$, ${ }^{* *} P<0.01$.

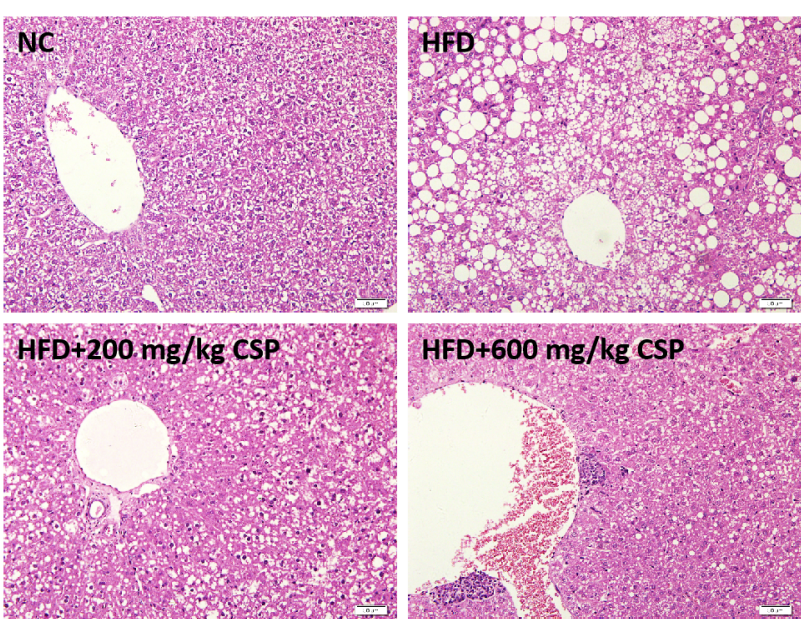

Fig. 4. HE staining of liver tissue of each group, scale $=50 \mu \mathrm{m}$.
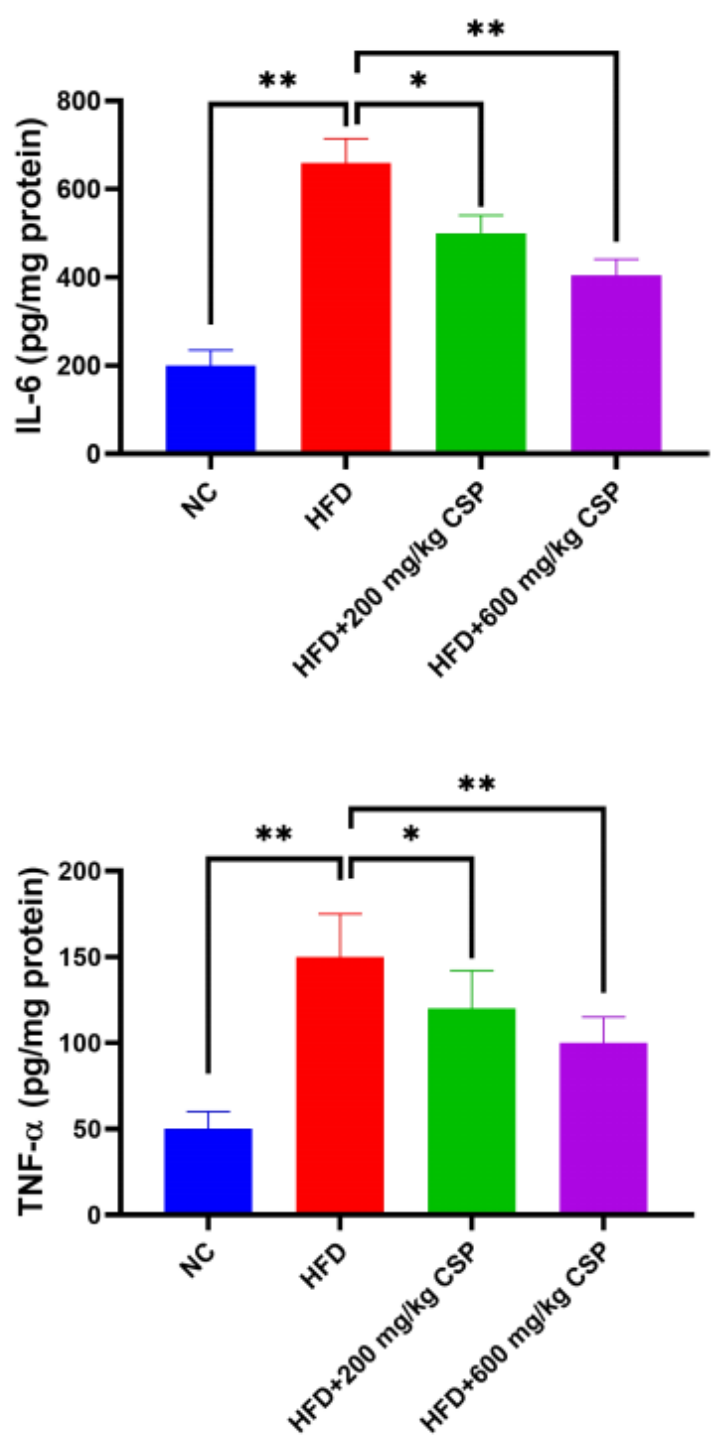

Fig. 5. CSP inhibits the inflammatory response of liver tissue induced by high fat diet. $* P<0.05, * * P<0.01$. 


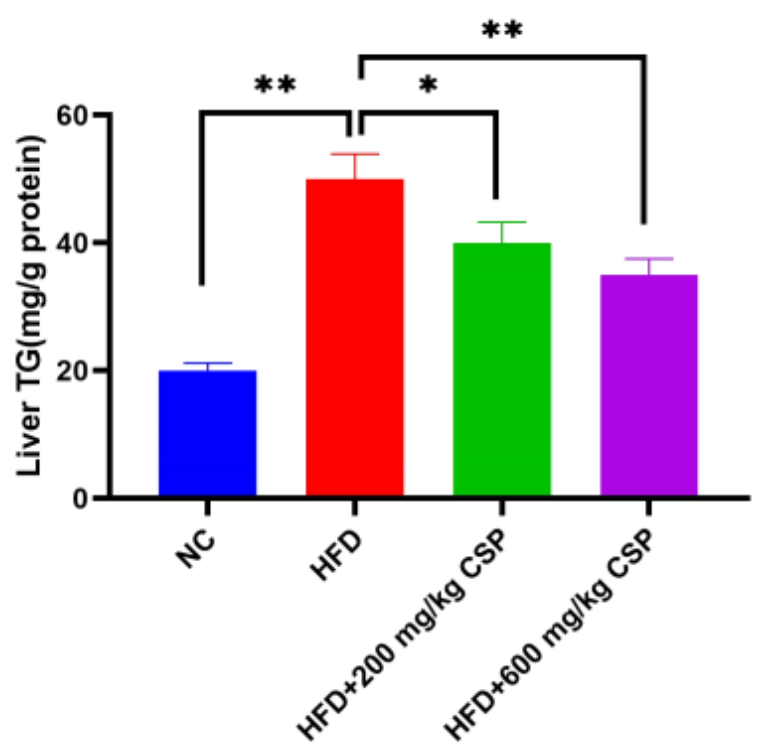

Fig. 6. The contents of TG in Liver of each group. $* P<0.05,{ }^{*} * P<0.01$.

\section{Conclusion}

In summary, HFD-induced NAFLD mice showed diffuse bullae steatosis of hepatocytes and abnormal excessive lipid deposition in steatotic hepatocytes accompanied by liver injury and liver inflammation. CSP can improve hepatocyte steatosis and lipid accumulation in hepatocytes induced by HFD and reduce liver inflammation, suggesting that CSP can be used as a therapeutic supplement against NAFLD.

\section{Acknowledgement}

This study was supported by the Science and Education Joint Project of Natural Science Foundation of Hunan Province (2020JJ707) and the Fund Project of Yueyang Social Science Federation (2019X93).

\section{References}

1. De Arka., Duseja Ajay.(2020). Natural History of Simple Steatosis or Nonalcoholic Fatty Liver. J Clin Exp Hepatol, 10(3), 255-262.

2. Klujszo Elzbieta H., Parcheta Piotr., Witkowska Anna B., Krecisz Beata.(2020). Non-alcoholic fatty liver disease in patients with psoriasis: therapeutic implications. Postepy Dermatol Alergol, 37(4), 468474.

3. Recena Aydos Leonardo., Aparecida do Amaral Luane., Serafim de Souza Roberta., Jacobowski Ana Cristina., Freitas Dos Santos Elisvânia., Rodrigues Macedo Maria Lígia.(2019). Nonalcoholic Fatty Liver Disease Induced by High-Fat Diet in C57bl/6 Models. Nutrients, 11(12).

4. Zhao Yan-Ci., Zhao Guo-Jun., Chen Ze., She ZhiGang., Cai Jingjing., Li Hongliang.(2020).
Nonalcoholic Fatty Liver Disease: An Emerging Driver of Hypertension. Hypertension, 75(2), 275284.

5. Kirrella Abeer A., Abdo Safaa E., El-Naggar Karima., Soliman Mohamed Mohamed., Aboelenin Salama Mostafa., Dawood Mahmoud A O., Saleh Ahmed A.(2021). Use of Corn Silk Meal in Broiler Diet: Effect on Growth Performance, Blood Biochemistry, Immunological Responses, and Growth-Related Gene Expression. Animals (Basel), 11(4).

6. Jia Yanan., Wang Yajie., Li Ruilin., Li Shuqin., Zhang Min., He Chengwei., Chen Haixia.(2021). The structural characteristic of acidic-hydrolyzed corn silk polysaccharides and its protection on the HO-injured intestinal epithelial cells. Food Chem, 356.

7. Jia Yanan., Gao Xudong., Xue Zihan., Wang Yajie., Lu Yangpeng., Zhang Min., Panichayupakaranant Pharkphoom., Chen Haixia.(2020). Characterization, antioxidant activities, and inhibition on $\alpha$ glucosidase activity of corn silk polysaccharides obtained by different extraction methods. Int J Biol Macromol 